\title{
The Role of Hypogammaglobulinemia in Childhood Asthma
}

\author{
Ozel Yuruker ${ }^{1,{ }^{*}}$, Burcin Sanlidag ${ }^{2}$, Murat Uncu $^{3}$ and Nerin Bahceciler ${ }^{4}$ \\ ${ }^{1}$ Division of Immunology and Allergy, Department of Clinical Biochemistry, Near East University, Nicosia, North Cyprus \\ ${ }^{2}$ Department of Pediatrics, Near East University, Nicosia, North Cyprus \\ ${ }^{3}$ Department of Clinical Biochemistry, Near East University, Nicosia, North Cyprus \\ ${ }^{4}$ Division of Pediatric Allergy, Department of Pediatrics, School of Medicine, Near East University, Nicosia, North Cyprus \\ "Corresponding author: Master of Science, Division of Immunology and Allergy, Near East University, Nicosia, North Cyprus. Tel: +90-3926751000, Email: \\ enver.kneebone@gmail.com
}

Received 2017 October 18; Revised 2018 February 19; Accepted 2018 April 03.

\begin{abstract}
Background: The relationship between hypogammaglobulinemia and wheezing in childhood has been previously revealed. Objectives: The study aimed to investigate co-morbid immune deficiency in unresponsive asthmatic children, characterize the type of immune deficiency, and determine any possible effect of immune deficiency treatment on the applied standard guideline of asthma treatment.

Methods: This was a prospective study conducted between January 2012 and December 2014. A total of 286 children whose serum immunoglobulin (Ig) levels had been analyzed were collected. Among those children, $125(\mathrm{~m} / \mathrm{f}=79: 46$, mean + -SD of age $=41.3+25.2$ months) were enrolled as they had uncontrolled moderate to persistent asthma. Those 125 children were categorized as the only asthma group and the asthma + hypogammaglobulinemia (HGG) group depending on their immunoglobulin concentrations.

Results: Seventy-six of the 125 children (60.8\%) had co-morbid hypogammaglobulinemia. Atopy was higher in the Asthma + HGG group $(\mathrm{P}=0.044)$. The most frequent co-morbid HGG was transient hypogammaglobulinemia of infancy (THGI) (46.1\%) and IgG subclass deficiency (32.9\%). Although the comparison of the percentage use of the inhaled corticosteroids (ICSs) showed no significant difference between the two groups at the initial evaluation, the dose of ICS significantly decreased only in the asthma + HGG group $(\mathrm{P}=0.017)$.

Conclusions: The majority of asthmatic children having symptoms despite appropriate guideline-based treatment may have a co-morbid immunological abnormality. Presented data demonstrated the necessity of immunological evaluation of uncontrolled asthmatic children to prevent long-term side effects of high-dose ICS, reduce the frequency and severity of asthma symptoms, and improve the quality of life.
\end{abstract}

Keywords: Allergy, Asthma, Atopy, Childhood, Hypogammaglobulinemia, Immunoglobulin, Transient Hypogammaglobulinemia of Infancy

\section{Background}

Asthma is an immunological disease that is characterized by persistent inflammation of the airways, bronchial hyperreactivity (BHR), recurrent wheezing, cough, and shortness of breath (1). It is common in children with different phenotypes that are characterized by the persistence or remittance of wheezing (transient early wheeze, non-atopic wheeze, and atopic-asthma) (2-4).

The treatments of asthma include allergen avoidance, pharmacological treatments, and allergen-specific immunotherapy (5). Corticosteroids are the most important class of medications used for the control of asthma symptoms as they have broad anti-inflammatory effects (6). Currently, the clinical approach to childhood asthma is based on the globally administered stepwise follow-up and treatment protocols, which recommend to increase the dose of inhaled corticosteroid(ICS) and/or add a variety of controller asthma medications for asthmatic children who are partially controlled $(6,7)$. In children, it is important to manage the treatment with minimum medication side effects (8).

It was previously reported that a significant number of children with recurrent wheezing had co-morbid hypogammaglobulinemia (HGG), highlighting the presence of a causal relationship between HGG and childhood wheezing in the general population (4, 5, 9-11). In one study, a $66.6 \% \operatorname{IgG}_{3}$ deficiency was reported among recurrent wheezers (5) while another study reported a 36\% HGG among children with asthma (4). Immunoglobulin (Ig) levels are normalized in some children at an earlier age, while some of them retain persistent low Ig levels (12). 
However, little is known about the importance of treating the co-morbid HGG alongside the asthmatic symptoms.

\section{Objectives}

The aim of this study was to investigate the co-morbid immune deficiency in asthmatic children who were unresponsive/partially responsive to the standard guideline treatment, characterize the type of immune deficiency, and determine any possible effect of immune deficiency treatment on the applied standard guideline of asthma treatment.

\section{Methods}

\subsection{Ethical Approval}

The Near East University Scientific Research Ethics Evaluation Board (YDU/2017/43-359) approved the study on January 18, 2017.

\subsection{Study Population}

A prospective study was conducted from January 2012 to December 2014 in the Division of Pediatric Allergy and Immunology at the Near East University Hospital, Cyprus, which is the only tertiary center for pediatric allergy and immunology in North Cyprus.

The medical records of patients with recurrent wheezing who had undergone a quantitative serum Ig analysis during the follow-up period were reviewed. Inclusion and exclusion criteria were as follows.

The inclusion criteria were children (i) with recurrent wheezing, cough, and/or croup who were unable to decrease inhaled steroid dose for control of symptoms (partially controlled) according to the guidelinerecommended treatment (7), (ii) who were investigated for immunodeficiency, (iii) who had a follow-up period of more than six months and, (iv) who did not receive allergen-specific immunotherapy.

The exclusion criteria were children (i) with only recurrent infections (13-15) and(ii) who had co-morbidities (such as gastroesophageal reflux, sinusitis, or cystic fibrosis).

The demographic, clinical, and laboratory findings of the children included in the study were collected from the hospital's database program retrospectively.

Information including gender, age, family history of immunodeficiency and allergy, follow-up period, remission of hypogammaglobulinemia, initial and final treatments, serum Ig levels, IgG subgroup levels, lymphocyte subset percentages, specific antibody responses, skin prick test, and allergen-specific IgE results had been recorded.

Asthmatic children were divided into two groups based on their serum Ig levels. HGG was defined as the serum Ig values of lower than two Standard Deviations of the mean for age and gender-matched controls (16).

\subsection{Follow-Up Period and Treatment}

According to the follow-up protocol established by the head of the Division of Pediatric Allergy and Immunology of Near East University Hospital, asthmatic children with recurrent wheezing, cough, and croup who partially respond or do not respond to the standard asthma medications and environmental precautions should be further evaluated in order to rule out co-morbidities including gastroesophageal reflux, sinusitis, cystic fibrosis, and immune deficiency diseases before accepting the patient as a more severe asthma case. Gastroesophageal reflux was ruled out based on the history of and unresponsiveness to anti-reflux treatment. Sinusitis was ruled out based on the history of revealing prolonged purulent nasal discharge, postnasal dripping, wet cough, and physical examinations. For cystic fibrosis, the sweat test was performed.

Next, the evaluation of the immune system was performed including the measurements of the serum Ig and IgG subclass levels. Further tests such as for determination of the lymphocyte subset percentages and specific antibody responses were performed if indicated by the clinician.

For the treatment of asthma, ICS was used as the first line medication. The dosage of ICS was adjusted according to the severity of symptoms based on the guideline recommendations10. Patients were controlled every two months in order to adjust the ICS dosage. All clinical follow-up and work-up throughout the study were performed by the same investigator.

\subsection{Immunologic and Other Laboratory Studies}

\subsubsection{Atopy}

a) Skin prick test

Skin prick test (SPT) was performed with $21 \mathrm{com}$ mon aero-allergens including Dermatophagoides farinae, Dermatophagoides pteronyssinus, Alternaria, Aspergillusmix, Penicillium mix, Candida albicans, Betulaceae, Aesculus hippo, Olea europaea, Plantago lanceolata, Artemisia vulgaris, Parietaria judaica, Secale cereale, Triticum vulgaris, Acacia dealbata, and a mixture of five grasses, feathers, cat hair, dog hair, and cockroach (Stallergenes, Antony, France). Histamine and saline were used as positive and negative controls, respectively. A drop of each allergen extract was placed on the volar surface of the left forearm and penetrated with a staller point. After 15 minutes, the wheal reaction was measured as the mean of the longest diameter and the diameter perpendicular to it. A wheal diameter of at least $3 \mathrm{~mm}$ greater than those of the negative control was considered positive. 
b) Allergen-specific IgE antibody test

Specific IgE antibodies of suspected or known allergens were studied from serum samples by using the radioallergosorbent test (RAST).

\subsection{Assessment of Immunoglobulin Levels}

Serum IgG, IgA, and IgM levels were measured by the turbidimetric method using Roche Cobas c311 and commercially available kits (Roche Diagnostics, Mannheim, Germany).

Serum IgE levels were measured by fully automated ELISA Roche Cobas 411 using commercially available kits (Roche Diagnostics, Mannheim, Germany).

The quantification of serum IgG subclasses $\left(\operatorname{IgG}_{1}, \operatorname{IgG}_{2}\right.$, and $\mathrm{IgG}_{3}$ ) was performed by nephelometry with commercially available kits. Results were evaluated according to the normal \pm 2 SD values based on the levels of healthy Turkish children according to age16. Values of lower than 2SD were accepted as low.

\subsection{Lymphocyte Subset Analysis}

Lymphocyte subpopulation; total lymphocytes (CD45), total T-cells (CD3), helper T-cells (CD3+/CD4+), cytotoxic Tcells (CD3+/CD8+), B-cells (CD19+), NK cells (CD16/56+), and Active T-cells (CD3/ anti-HLA DR) were analyzed via flow cytometry (BD FACS Calibur, BD Biosciences, San Jose, CA, USA).

For each patient, blood samples collected in heparinized tubes were used in six different tubes labeled according to markers used. The first tube was the isotype control. $10 \mu \mathrm{L}$ of the surface marker and $100 \mu \mathrm{L}$ of peripheral blood were mixed and incubated for 20 minutes at room temperature (RT). At the end of incubation, $2 \mathrm{~mL}$ of lysing solution was added to each tube for the elimination of red blood cells and the tubes were incubated for 10 minutes at RT. After incubation, the tubes were centrifuged for seven minutes at $1500 \mathrm{~g}$. The supernatants were discarded and 2 $\mathrm{mL}$ of cell wash solution was added to each tube. The tubes were then centrifuged for seven minutes at $1500 \mathrm{~g}$. Supernatants were then discarded and $500 \mu \mathrm{L}$ of cell wash solution was added to all tubes for analysis.

During analysis, lymphocytes were gated according to the CD 45 surface marker. Then, the percentages of all markers were detected. Results were evaluated according to the normal \pm 2 SD values based on age for Turkish children (17). Values of lower than 2 SD were accepted as low.

\subsection{Antigen-Specific Antibody Response}

Antigen-specific antibody responses were evaluated by anti-tetanus toxoid IgG antibody level, EuroImmune IgG ELISA, Pneumococcus IgG, ELIZEN/ZenTech (catalogue no:EDG-96), and anti- Hbs, fully automated ELISA Roche Cobas
411 (catalogue no: El 2060-9601 G), with commercially available kits (Roche Diagnostics, Mannheim, Germany).

All the equipment used for the analyses of Ig levels, lymphocyte subsets, and antigen-specific antibody responses is calibrated routinely in the Laboratory of the Near East University Hospital. In addition, control samples are routinely used prior to the analysis of the patients' serum samples.

\subsection{Statistical Analysis}

Statistical analyses were performed using the IBM SPSS Statistics for Windows, version 20.0 (IBM Corp., Armonk, N.Y. USA). Descriptive statistics were expressed as mean (SD), median, and range. Prevalence rates were expressed as percentages. Chi-square, Kruskal-Wallis, and Mann Whitney U tests were used for analyses. Pvalues of less than 0.05 were considered statistically significant.

\section{Results}

The medical records of 286 children whose serum Ig levels had been previously analyzed were overviewed. Among those 286 children, 125 met the inclusion criteria, and they were enrolled in our study. The remaining 161 children were excluded from the study. One hundred forty had only recurrent infections without any asthmatic symptoms while three were excluded due to the loss of followup and 18 had co-morbid diseases (except for HGG), complicating the asthma course. Asthma patients with and without antibody deficiency were compared based on demographic characteristics, atopy, family history, initial and end of follow-up, immunological test results, and ICS consumption (Figure 1). Further clinical evaluation prompted the analysis of serum IgG subclasses of 63 patients and lymphocyte subset analysis of 25 patients.

\subsection{Demographic Characteristics of Patients}

Demographic characteristics of those 125 children who were included are presented in Table 1.

The age range and the male percentage of the125 patients who were included in our study were 5 - 137 months and $63.2 \%$, respectively. The family history of allergic diseases, primary immune deficiency (PID) diseases, and consanguineous marriage of parents were found to be $54.9 \%$, $4.9 \%$, and $2.5 \%$, respectively. Although the reasons for the admission of those 125 children to the Pediatric Allergy and Immunology Division were recurrent wheezing (10.4\%), recurrent cough/croup (10.4\%), both of them (71.2\%), and any of those plus recurrent infections ( $8 \%$ ), $60.8 \%$ of them were diagnosed as having hypogammaglobulinemia. In other words, the majority of patients (92\%) admitted with 


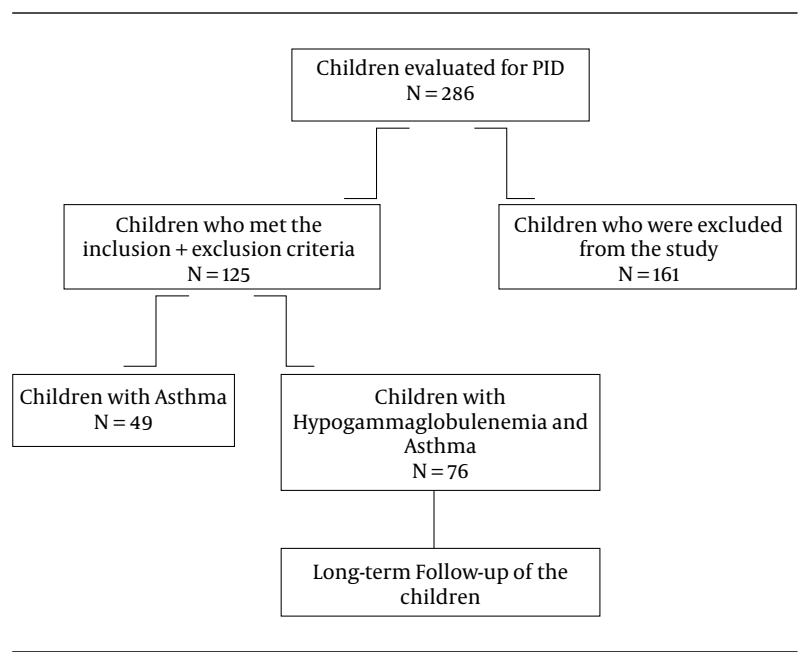

Figure 1. The scheme of the study design. Among 286 patients whose Ig titers were analyzed, 125 were included in the study and 161 were excluded. 49 out of 125 were asthmatic children and 76 of 125 were asthmatic children with hypogammaglobulinemia. PID, Primary Immune Deficiency.

\begin{tabular}{|c|c|}
\hline Characteristics & Values $^{\mathbf{b}}$ \\
\hline Age (months) (min-max) & $(5-137)(41.3 \pm 25.2)$ \\
\hline Gender (male/female) & $79 / 46(63.2 / 36.8)$ \\
\hline \multicolumn{2}{|l|}{ Family history } \\
\hline Allergy & $67(54.9)$ \\
\hline PID disease & $6(4.9)$ \\
\hline Consanguineous marriage & $3(2.5)$ \\
\hline Diagnosis of PID & $76(60.8)$ \\
\hline \multicolumn{2}{|l|}{ Reason for admission } \\
\hline Recurrent wheezing & $13(10.4)$ \\
\hline Recurrent cough/croup & $13(10.4)$ \\
\hline Both & $89(71.2)$ \\
\hline Any of above +recurrent infection & $10(8.0)$ \\
\hline Follow-up period (min-max) & $(2-48)(12.1 \pm 12.28)$ \\
\hline Atopy $^{\mathrm{a}}$ & $55(67.1)$ \\
\hline
\end{tabular}

asthma-like symptoms while only $8 \%$ of them had a history of a recurrent infection in addition to asthmatic symptoms.

\subsection{Comparison of Patients with and Without $H G G$}

When children with and without a hypogammaglobulinemia were compared based on age, gender, admission complaints, family history of allergy, immune deficiency, and consanguineous marriage, no statistically significant differences were detected. On the other hand, serum total IgM, IgG, and IgG $\mathrm{G}_{3}$ were statistically significantly lower in the hypogammaglobulinemia group $(\mathrm{P}=0.016, \mathrm{P}<$ 0.0001 , and $\mathrm{P}<0.0001$, respectively). Although there was no significant difference in the serum total IgE levels between the asthmatic children with and without hypogammaglobulinemia $(P=0.638)$, the asthmatic children with hypogammaglobulinemia were significantly more atopic $(\mathrm{P}=0.044)$. The characteristics of asthmatic children with and without hypogammaglobulinemia are presented in Table 2 and Figure 2A - 2E.

\subsection{Characteristics of HGG Patients}

The most frequently diagnosed immune deficiency disease among the asthmatic children with hypogammaglobulinemia was transient hypogammaglobulinemia of infancy (THGI) (46\%), followed by IgG subclass deficiency (32.9\%). The distribution of immune deficiency diseases is presented in Table 3.

Among the 125 children evaluated, 76 had co-morbid hypogammaglobulinemia. Of those 76 children, 39 children had demonstrated to have low IgG, 14 had low IgA, 21 had low IgM, and 32 had low IgG 3 levels. A high IgE level was detected in 32 out of 76 PID children, the majority of which being diagnosed as IgG subclass deficiency.

The lymphocyte subset analysis results were normal for all patients with hypogammaglobulinemia except for three patients diagnosed as CVID, including one with low $\mathrm{CD} 4$, one with low CD19, and one with low CD8 levels (Table 4).

Specific antibody responses including Tetanus (one patient), pneumococcus (one patient), and hepatitis B (two patients) were low in CVID patients. In addition, the isohemagglutinin antibody response was detected low in three CVID patients (data not shown).

\subsection{Treatment}

Asthmatic children with and without hypogammaglobulinemia were also compared based on their "asthma" and "HGG" treatments. No statistically significant differences were observed between the groups at enrolment based on the asthma medications (ICS and /or montelukast-Na) needed for control of asthma symptoms. Asthmatic children with co-morbid hypogammaglobulinemia received additional treatments such as prophylactic antibiotics or IVIG plus prophylactic antibiotics. A decision on treatment was made based on the patients' clinical conditions. In case of severe and recurrent infections or secondary complications due to infections, IVIG was initiated. For moderate recurrent infections, prophylactic antibiotic treatment was prescribed. Those with mild recurrent infections such as upper respiratory tract infections 


\begin{tabular}{|c|c|c|c|}
\hline & Asthma + HGG & Asthma & P Value \\
\hline Gender (male) & $50 / 26(65.8 / 34.2)$ & $29 / 20(59.2 / 40.8)$ & 0.569 \\
\hline Admission complaints & & & 0.874 \\
\hline Recurrent wheezing & $7(9.2)$ & $6(12.2)$ & \\
\hline Recurrent cough/croup & $7(9.2)$ & $6(12.2)$ & \\
\hline Both & $56(73.7)$ & $33(67.3)$ & \\
\hline Any of above + recurrent infection & $6(7.9)$ & $4(8.2)$ & \\
\hline \multicolumn{4}{|l|}{ Family history } \\
\hline Allergy & $39(53.4)$ & $28(57.1)$ & 0.686 \\
\hline PID & $6(8.2)$ & $0(0)$ & $0.040^{\mathrm{b}}$ \\
\hline Consanguineous marriage & $3(41.1)$ & $0(0)$ & 0.151 \\
\hline
\end{tabular}

\begin{tabular}{lc}
\hline \multicolumn{2}{l}{ Table 3. Distribution of Diagnosed Immune Deficiency Diseases } \\
\hline Immune Deficiencies & Values $^{\mathbf{a}}$ \\
\hline THGI & $35(46.1)$ \\
\hline IgG subclass deficiency & $25(32.9)$ \\
\hline IgA deficiency & $1(1.3)$ \\
\hline CVID & $7(9.2)$ \\
\hline IgM deficiency & $6(7.9)$ \\
\hline PHGI & $1(1.3)$ \\
\hline Others & $1(1.3)$ \\
\hline
\end{tabular}

Abbreviations: CVID, common variable immune deficiency; PHGI, protracted hypogammaglobulinemia of infancy; THGI, transient hypogammaglobulinemia of infancy.

${ }^{a}$ Values are expressed as No. (\%).

were followed up without any medication for hypogammaglobulinemia17 (Table 5).

\subsection{Use of ICS}

The two groups of patients were compared based on the mean daily dose of ICS needed to control symptoms at enrolment, at the end of the study, and within both groups. The results revealed that although the dose of ICS decreased in both groups, the dose of ICS statistically significantly decreased in the asthma+hypogammaglobulinemia group $(P=0.024)$, whereas there was no significant difference in the only asthma group $(\mathrm{P}=0.068)$ (Figure 3 ).

\subsection{Use of Trim-Sulf or IVIG}

Within the asthma+hypogammaglobulinemia group of 70 patients, 28 (38.3\%) were under prophylaxis of TrimSulf. Two of them (2.7\%) were given both IVIG and Trim-Sulf.

\section{Discussion}

In the current study, we demonstrated that among 125 asthmatic children under treatment with ICS and/or Montelukast-sodium with partial response to medications, 76 (60.8\%) demonstrated to have a co-morbid hypogammaglobulinemia disease. Interestingly, atopy was higher in asthma + hypogammaglobulinemia group than in the only asthma group, and antibody levels were lower in those with hypogammaglobulinemia. The most frequent co-morbid hypogammaglobulinemia diseases were found to be THGI and IgG subclass deficiencies.

The daily dose of ICS for asthma control in the children with asthma + co-morbid hypogammaglobulinemia decreased significantly after the addition of appropriate treatment for hypogammaglobulinemia. Although the comparison of the percentage use of ICSs showed no significant difference between the two groups at the initial evaluation, the dose of ICS significantly decreased in the asthma+ hypogammaglobulinemia group $(\mathrm{P}=0.024)$, whereas there was no significant difference in the only asthma group $(\mathrm{P}=0.068)$ at the end of the follow-up period. It was thought that additional immune prophylaxis prevented recurrent infections that triggered the asthma symptoms in children with co-morbid hypogammaglobulinemia. This probably helped the decreased percentage use of ICSs throughout the treatment period.

Little is known about the characteristics of asthma children with HGG. In a study that was performed in adults, the prevalence of HGG in adult asthmatic patients was reported as 25\%18 while in our study, the prevalence of HGG in pediatric asthma patients was detected as $60.8 \%$, and 35 of them were diagnosed as THGI (28\%). After the exclusion of the THGI patients, the prevalence was re-calculated at $32 \%$, 
A

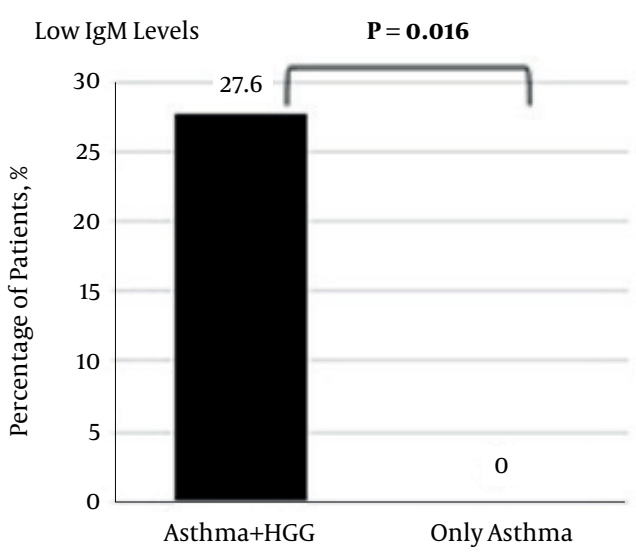

C

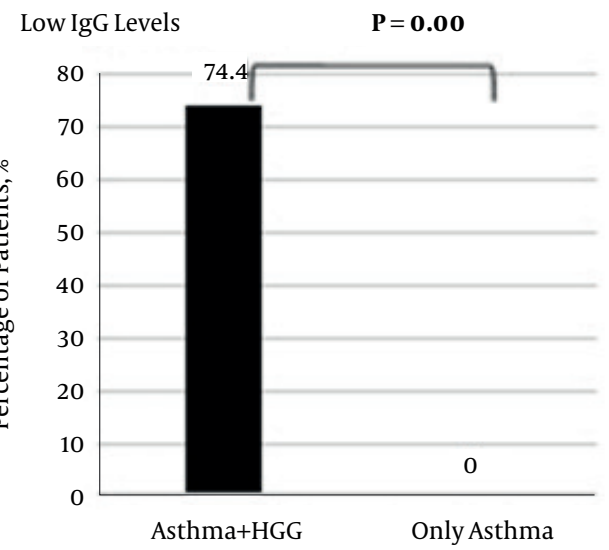

B

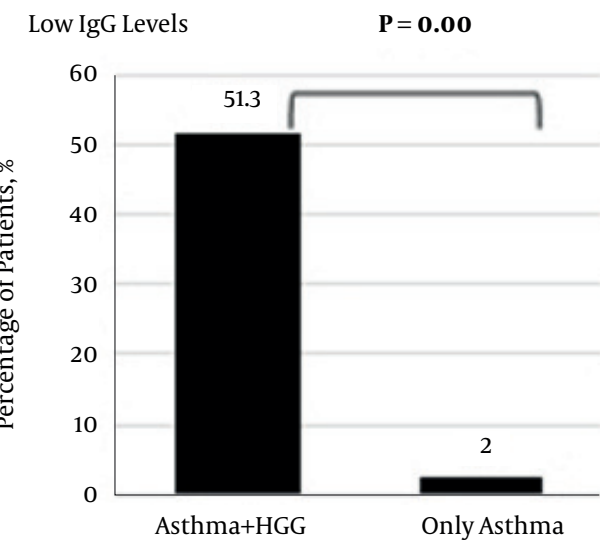

D

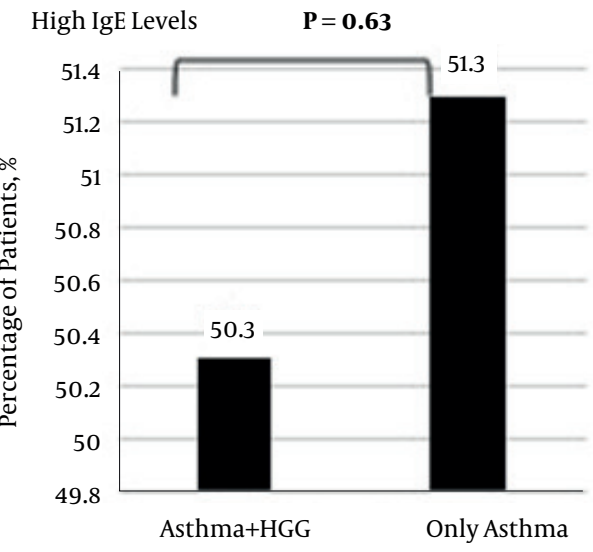

$\mathbf{E}$

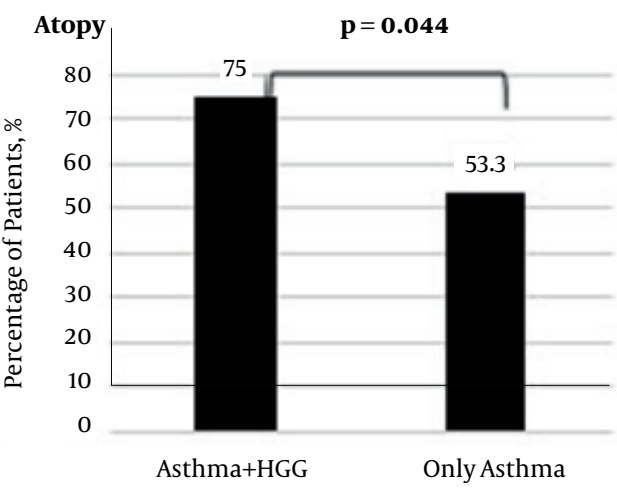

Figure 2. The comparison of Ig levels and presence of atopy in both groups: A-C, the percentages of low IgM, IgG, and IgG that were more frequent in the asthma + Hypogam maglobulinemia (HGG) group, D, serum IgE levels of the asthmatic patients with and without HGG that were the same, E, the comparison of atopic status of asthmatic children with and without HGG that revealed significantly more atopic sensitization in those who had asthma + HGG.

which is in accordance with the results of the adult study (18).

It is difficult to compare our study with previous inves- tigations due to the heterogeneity of age groups and the severity of asthmatic children in other studies published to date. In the study of Oner et al. all children with re- 


\begin{tabular}{|c|c|c|c|c|c|c|c|c|}
\hline & $\begin{array}{c}\text { THG of } \\
\text { Infancy }(n= \\
35)\end{array}$ & $\begin{array}{l}\text { IgG Subclass } \\
\text { Deficiency (n } \\
=25)\end{array}$ & $\begin{array}{l}\text { IgA Deficiency } \\
\qquad(\mathbf{n}=\mathbf{1})\end{array}$ & $\operatorname{CVID}(\mathbf{n}=7)$ & $\begin{array}{c}\text { IgM } \\
\text { Deficiency (n } \\
=6)\end{array}$ & $\begin{array}{c}\text { PHG of } \\
\text { Infancy }(n=1)\end{array}$ & Others $(n=1)$ & $\begin{array}{l}\text { Total Number } \\
\text { of Patients ( } \\
=76)\end{array}$ \\
\hline Low IgG & $33(84.6)$ & $1(2.6)$ & $0(0)$ & $4(10.3)$ & $0(0)$ & $1(2.6)$ & $0(0)$ & 39 \\
\hline Low IgA & $8(57.1)$ & $0(0)$ & $1(7.1)$ & $5(35.7)$ & $0(0)$ & $1(4.8)$ & $0(0)$ & 14 \\
\hline Low IgM & $7(33.3)$ & $2(9.2)$ & $0(0)$ & $5(23.8)$ & $6(28.6)$ & $1(4.8)$ & $0(0)$ & 21 \\
\hline Low IgG1 & $3(42.9)$ & $2(28.6)$ & $0(0)$ & $2(28.6)$ & $0(0)$ & $0(0)$ & $0(0)$ & 7 \\
\hline Low IgG2 & $1(100)$ & $0(0)$ & $0(0)$ & $0(0)$ & $0(0)$ & $0(0)$ & $0(0)$ & 1 \\
\hline Low IgG3 & $7(21.9)$ & $22(68.8)$ & $0(0)$ & $1(3.1)$ & $1(3.1)$ & $1(3.1)$ & $0(0)$ & 32 \\
\hline High IgE & $10(33)$ & $14(46.7)$ & $0(0)$ & $3(10)$ & $3(10)$ & $0(0)$ & $0(0)$ & 30 \\
\hline Low CD45 & $0(0)$ & $0(0)$ & $0(0)$ & $0(0)$ & $0(0)$ & $0(0)$ & $0(0)$ & 22 \\
\hline Low CD19 & $0(0)$ & $0(0)$ & $0(0)$ & $1(50)$ & $0(0)$ & $0(0)$ & $1(50)$ & 2 \\
\hline Low CD3 & $0(0)$ & $0(0)$ & $0(0)$ & $0(0)$ & $0(0)$ & $1(100)$ & $0(0)$ & 1 \\
\hline Low CD4 & $0(0)$ & $0(0)$ & $0(0)$ & $1(100)$ & $0(0)$ & $0(0)$ & $0(0)$ & 1 \\
\hline Low CD8 & $0(0)$ & $0(0)$ & $0(0)$ & $1(100)$ & $0(0)$ & $0(0)$ & $0(0)$ & 1 \\
\hline Low CD16/56 & $2(100)$ & $0(0)$ & $0(0)$ & $0(0)$ & $0(0)$ & $0(0)$ & $0(0)$ & 2 \\
\hline Low Active T & $4(80)$ & $0(0)$ & $0(0)$ & $0(0)$ & $0(0)$ & $1(20)$ & $0(0)$ & 5 \\
\hline
\end{tabular}

Abbreviations: PHG, protracted hypogammaglobulinemia; THG, transient hypogammaglobulinemia.

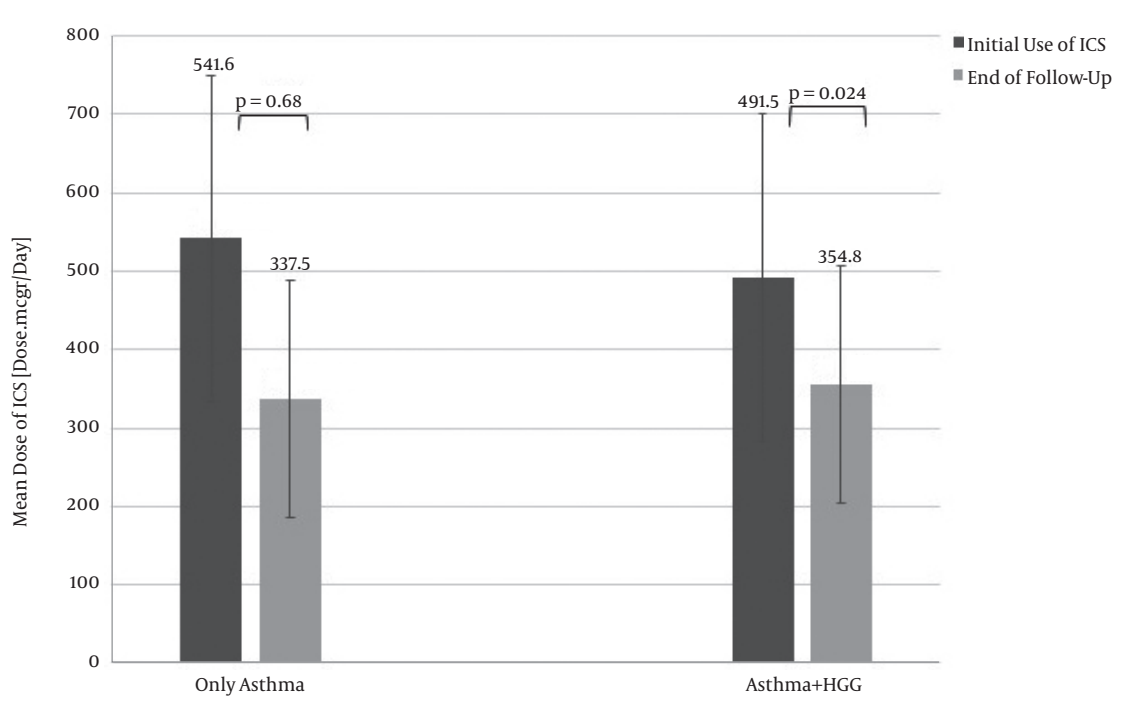

Figure 3. The comparison of the mean daily dose of ICS between and within groups. The mean dose of ICSs decreased in both groups; however, according to P values, it was statistically significant in the asthma+ HGG group

current wheezing regardless of severity and/or response to treatment who were 9 - 24 months and $2-6$ years of age were included and compared with healthy controls based on Ig and IgG subgroup levels. They demonstrated that recurrent wheezy children in the 2 - 6 years age group had significantly lower $\operatorname{IgG}_{3}$ levels compared to their healthy counterparts (10).

Another study included all children with asthma un- der follow-up, regardless of the severity and studied immunoglobulin levels. Then, they compared the features of asthmatic children having normal Ig levels and hypogammaglobulinemia. Their results revealed that children with hypogammaglobulinemia had an earlier onset of the disease, lower rates of atopy, and earlier clinical improvement compared to the group having normogammaglobulinemia (4). Another study revealed that the long-term follow- 


\begin{tabular}{lccc}
\hline \multicolumn{4}{l}{ Table 5. Distribution of Treatments That Were Given to Patients in Both Groups ${ }^{\mathrm{a}}$} \\
\hline & Asthma + HGG & Only Asthma & P Value \\
\hline Asthma treatment & & & \\
\hline ICS & $46(62.9)$ & $23(52.5)$ & 0.767 \\
\hline Montelukast-Na & $5(6.8)$ & $8(18.2)$ & 0.107 \\
PID treatment & & & \\
\hline Trim - Sulf & $28(38.3)$ & $0(0)$ & - \\
\hline IVIG + Trim - Sulf & $2(2.7)$ & $0(0)$ & \\
\hline
\end{tabular}

Abbreviation: HGG: hypogammaglobulinemia; ICS: inhaled corticosteroid; IVIG: intravenous immunoglobulin; Trim - Sulf: trimethoprim and sulfamethoxazole.

${ }^{\text {a }}$ Values are expressed as No. (\%)

up of children with low IgG $\mathrm{G}_{3}$ levels could be susceptible to asthma or other allergic diseases (19). Although those studies reported a relationship between low Ig and IgG subclass levels and recurrent wheezing, they did not differentiate between clinical phenotypes and severity of asthmatic children. Therefore, the importance and strategies that should be taken in the treatment of children with different asthma severities cannot be deduced. On the other hand, in our study, children of all age groups having mild to moderate persistent asthma, who were partly responsive or unresponsive to standard asthma treatment, were included. Our results suggested that before increasing the dose of ICS or adding other asthma treatments, the nonresponder children should be evaluated for co-morbid hypogammaglobulinemia.

In addition, the results of all those three studies demonstrated that hypogammaglobulinemia in children might be present with isolated asthma symptoms. The first study reported a $66.6 \% \operatorname{IgG}_{3}$ deficiency among recurrent wheezers (5) while the study of Baris et al. reported a $36 \%$ HGG rate among children with asthma (4). In accordance with those results, $60.8 \%$ of our group had co-morbid HGG. The current study was performed in the pediatric AllergyImmunology Division of a University Hospital. This may be the reason for the high HGG rates in asthmatic children. Based on those high HGG rates, in children who have insufficient response to the guideline-based asthma treatment plans during the long-term pediatric asthma followup, underlying immune deficiency should be considered. There are limited published data in the literature on this subject.

In our study, serum total IgM, IgG, and IgG $G_{3}$ levels were statistically significantly lower in the HGG + asthma group $(\mathrm{P}=0.016, \mathrm{P}=0.000$, and $\mathrm{P}=0.000$, respectively) as expected. Atopy was higher in the asthma+HGG group, in contrast to a study performed previously (4), showing that low Ig levels can be detected in a significant proportion of children with asthma, especially in non-atopic cases. It was previously shown that the lower rates of atopy were present in asthmatic children with PID disease (17.9\%) with earlier clinical improvement accompanied by the discontinuation of ICS when compared to the children with normal Ig levels (4). However, we have shown that there is no strict association between non-atopy and low Ig level in asthmatic children.

In the study of Baris et al., the patients were classified depending on the presence of HGG (4). However, the results of further immunological evaluation were not reported. Therefore, making a comparison based on the type of immune deficiency is not possible. In our study, the most frequently diagnosed HGG among the asthmatic children with low Igs was THGI (46\%), followed by an IgG Iefi- $_{3}$. ciency (32.9\%).

In accordance with the results of Baris et al., the addition of appropriate treatment for HGG in children with asthma + HGG resulted in an earlier significant decrease in the daily consumption of ICSs (4). Currently, there are limited published data on HGG as an underlying cause of uncontrolled childhood asthma. Our study underlines the importance of the immunological evaluation in asthmatic children unresponsive to the standard treatment.

It is well known that the symptoms of children with only asthma improve by age, especially in those with nonatopic disease $(3,4)$. One of the limitations of the study was that it was not possible to include a control group of children with asthma + HGG without treatment for HGG due to ethical issues. The diagnosis of any HGG in children prompts an appropriate treatment in order to prevent the long-term irreversible changes in airways such as bronchiectasis $(20,21)$. In addition, another limitation was that an additional control group of asthmatic children who were responsive to the standard treatment was not included and could not be compared as generally, further immunological tests are not a necessity for responsive children, and they are not a routine investigation in our department.

In conclusion, the current study demonstrated that the majority of asthmatic children who continue to have symptoms despite appropriate guideline-based treatment might have a co-morbid immunological abnormality. The treatment of co-morbid hypogammaglobulinemia improves asthma control and enables the reduction of ICS doses in those children. These results underline the necessity of immunological evaluations in uncontrolled childhood asthma in order to prevent the long-term side effects of high-dose ICS, and to prevent the frequency and severity of asthma symptoms, and iii) decrease the morbidity and improve the quality of life. 


\section{Footnotes}

Authors' Contribution: Ozel Yuruker did laboratory tests, did the data collection from the university database, helped the statistical analysis, and wrote the manuscript. Burcin Sanlıdag; did the patient follow-up, did the data collection from the database programme, contributed the writing of the manuscript Murat Uncu; helped the laboratory tests and interpreting the laboratory test results. Nerin Baheciler; was the mentor and advisor of the study. She examined the patients and did the follow-up of patients, did the statistical analysis of the data collected and advise on writing of the manuscript.

Financial Disclosure: No financial interests or financial conflicts.

Funding/Support: No funding was taken for the study.

\section{References}

1. Martinez FD, Vercelli D. Asthma. Lancet. 2013;382(9901):1360-72. doi: 10.1016/S0140-6736(13)61536-6. [PubMed: 24041942].

2. Stein RT, Martinez FD. Asthma phenotypes in childhood: Lessons from an epidemiological approach. Paediatr Respir Rev. 2004;5(2):15561. doi:10.1016/j.prrv.2004.01.007. [PubMed:15135126].

3. Sly PD, Boner AL, Bjorksten B, Bush A, Custovic A, Eigenmann PA, et al. Early identification of atopy in the prediction of persistent asthma in children. Lancet. 2008;372(9643):1100-6. doi: 10.1016/S0140-6736(08)61451-8. [PubMed: 18805338]. [PubMed Central: PMC4440493].

4. Baris S, Karakoc-Aydiner E, Ozen A, Ozdemir C, Bahceciler NN, Barlan IB. Serum immunoglobulin levels as a predictive factor for a better outcome of non-atopic childhood asthma. Pediatr Allergy Immunol. 2011;22(3):298-304. doi: 10.1111/j.1399-3038.2010.01105.x. [PubMed: 20961339].

5. Alvaro M, Sancha J, Larramona H, Lucas JM, Mesa M, Tabar AI, et al. Allergen-specific immunotherapy: Update on immunological mechanisms. Allergol Immunopathol (Madr). 2013;41(4):265-72. doi: 10.1016/j.aller.2012.07.018. [PubMed: 23332741].

6. Adelman DC, Casale TB, Corren J. Manual of allergy and immunology. 5th ed. Philadelphia: Lippincott Williams and Wilkins; 2012.

7. Global Initiative for Asthma. Pocket guide for asthma management and prevention for children 5 years and younger. 2015. Available from: http: //www.ginasthma.org.
8. van Aalderen WM. Childhood asthma: Diagnosis and treatment. Scientifica (Cairo). 2012;2012:674204. doi: 10.6064/2012/674204. [PubMed: 24278725]. [PubMed Central: PMC3820621].

9. Szczawinska-Poplonyk A. An overlapping syndrome of allergy and immune deficiency in children. J Allergy (Cairo). 2012;2012:658279. doi: 10.1155/2012/658279. [PubMed: 21918651]. [PubMed Central: PMC3171763].

10. Oner AF, Caksen H, Celik A, Cesur Y, Uner A, Arslan S. Serum immunoglobulins and immunoglobulin $G$ subclasses with recurrent wheezing. Indian J Pediatr. 2000;67(12):861-4. [PubMed: 11262982].

11. Karaman O, Uguz A, Uzuner N. IgG subclasses in wheezing infants. Indian J Pediatr. 1999;66(3):345-9. [PubMed:10798081].

12. Ozen A, Baris S, Karakoc-Aydiner E, Ozdemir C, Bahceciler NN, Barlan IB. Outcome of hypogammaglobulinemia in children: Immunoglobulin levels as predictors. Clin Immunol. 2010;137(3):374-83. doi: 10.1016/j.clim.2010.08.010. [PubMed: 20851686].

13. Paul ME, Shearer WT. The child who has recurrent infection. Immunol Aller Clin North Am. 1999;19(2):423-36. doi: 10.1016/s08898561(05)70097-7.

14. Woroniecka M, Ballow M. Office evaluation of children with recurrent infection. Pediatr Clin North Am. 2000;47(6):1211-24. [PubMed: 11130993].

15. Kliegman R, Nelson WE. Evaluation of suspected immunodeficiency. In: Kliegman RM, Stanton BF, St Geme JW, Schor NF, Behrman RE, editors. Nelson Textbook of Pediatrics. Elsevier/Saunders; 2011.

16. Aksu G, Genel F, Koturoglu G, Kurugol Z, Kutukculer N. Serum immunoglobulin (IgG, IgM, IgA) and IgG subclass concentrations in healthy children: A study using nephelometric technique. Turk J Pediatr. 2006;48(1):19-24. [PubMed: 16562781].

17. Ikinciogullari A, Kendirli T, Dogu F, Egin Y, Reisli I, Cin S, et al. Peripheral blood lymphocyte subsets in healthy Turkish children. Turk J Pediatr. 2004;46(2):125-30. [PubMed: 15214740].

18. Dupin C, Marchand-Adam S, Favelle O, Costes R, Gatault P, Diot P, et al. Asthma and hypogammaglobulinemia: An asthma phenotype with low type 2 inflammation. J Clin Immunol. 2016;36(8):810-7. doi: 10.1007/s10875-016-0335-9. [PubMed: 27714565].

19. Ones U, Guler N, Somer A, Salman N, Yalcin I. Low immunoglobulin G3 levels in wheezy children. Acta Paediatr. 1998;87(4):368-70. [PubMed: 9628287].

20. Spickett G. Oxford handbook of clinical immunology and allergy. 3rd ed. Oxford: Oxford University Press; 2013. p. 1-33. doi: 10.1093/med/9780199603244.001.0001.

21. Moise A, Nedelcu FD, Toader MA, Sora SM, Tica A, Ferastraoaru DE, et al. Primary immunodeficiencies of the B lymphocyte. J Med Life. 2010;3(1):60-3. [PubMed: 20302197]. [PubMed Central: PMC3019034]. 